\title{
Signet-ring cell melanoma with sentinel lymph node metastasis: A case report with immunohistochemical analysis and review of the clinicopathological features
}

\author{
MITSUAKI ISHIDA, MUNEO IWAI, KEIKO YOSHIDA, AKIKO KAGOTANI and HIDETOSHI OKABE \\ Department of Clinical Laboratory Medicine and Division of Diagnostic Pathology, \\ Shiga University of Medical Science, Otsu, Shiga 520-2192, Japan
}

Received April 11, 2013; Accepted October 22, 2013

DOI: $10.3892 / \mathrm{ol} .2013 .1669$

\begin{abstract}
Signet-ring cell melanoma is an extremely rare variant of malignant melanoma. A 68-year-old male presented with a black nodule on the left thigh. Histopathological examination revealed proliferation of sheet-like or variable-sized nests of atypical melanocytes. Neoplastic cells showing signet-ring cell appearance, characterized by the presence of eccentrically located enlarged nuclei and abundant pale cytoplasm, were also present. Immunohistochemically, the tumor cells were positive for S-100 protein, vimentin and Melan-A. Moreover, mammalian target of rapamycin (mTOR) pathway proteins were diffusely expressed. The current case report presents the 21st reported case of signet-ring cell melanoma. Analyses of the clinicopathological features revealed that this disease commonly affects middle-aged males and the presence of metastatic signet-ring cell melanoma with an unknown primary tumor. Immunohistochemical analyses of melanocytic markers have been useful for establishing the diagnosis of this type of disease, however, HMB-45 is occasionally found to be negative. In addition, the present case report is the first to analyze the expression of mTOR pathway proteins, which are central proteins involved in carcinogenesis and its inhibitor has been proposed as a therapeutic target for various types of tumor. Therefore, the mTOR inhibitor may also be a potential candidate for the treatment of this type of tumor.
\end{abstract}

\section{Introduction}

Malignant melanoma occasionally shows a variety of cytomorphological and architectural features, including balloon,

Correspondence to: Dr Mitsuaki Ishida, Department of Clinical Laboratory Medicine and Division of Diagnostic Pathology, Shiga University of Medical Science, Tsukinowa-cho, Seta, Otsu, Shiga 520-2192, Japan

E-mail: mitsuaki@belle.shiga-med.ac.jp

Key words: signet-ring cell melanoma, intermediate filament, mTOR pathway rhabdoid, small, myxoid, adenoid (pseudoglandular) and clear cell types (1). Signet-ring cell melanoma is one of the rarest histopathological variants of malignant melanoma, characterized histopathologically by the presence of tumor cells in which the nucleus is compressed to the cellular periphery, appearing as signet-rings (2). This variant was initially described by Sheibani and Battifora in 1988 (3) and since then, 20 cases have been reported in English literature (2-15). The current case report presents an additional case of signet-ring cell melanoma with sentinel lymph node metastasis, analyses of the immunohistochemical expression profiles of the intermediate filaments and mammalian target of rapamycin (mTOR) pathway proteins and review of the clinicopathological features of this extremely rare variant of malignant melanoma.

\section{Case report}

Patient presentation. A 68-year-old male without a past history of malignant melanoma presented with a gradually enlarged black nodule in the left thigh. Physical examination revealed a relatively well-circumscribed nodule, measuring 25x20 mm in diameter, with uneven pigmentation in the patients thigh. Systemic surveillance failed to identify additional tumorous lesions other than the tumor in the left thigh. Total resection of the nodule was performed and subsequently, dissection of the sentinel lymph node. Written informed consent was obtained from the patient.

Materials andmethods. The formalin-fixed,paraffin-embedded tissue blocks of the resected skin specimen and lymph nodes were cut into 3- $\mu \mathrm{m}$-thick sections, deparaffinized and rehydrated. Each section was stained with hematoxylin and eosin and then used for immunostaining. Immunohistochemical analyses were performed using an autostainer (BenchMark XT system; Ventana Medical System, Tucson, AZ, USA) according to the manufacturer's instructions. The following primary antibodies were used: Mouse monoclonal antibodies against $\alpha$-internexin (2E3; Lab Vision Corp., Fremont, CA, USA), cytokeratin (AE1/AE3 and CAM5.2; DakoCytomation, Glostrup, Denmark and Becton-Dickinson, Franklin Lakes, NJ, USA, respectively), glial fibrillary acid protein (GFAP; 6F2; DakoCytomation), HMB-45 (Novocastra Laboratories, 
Table I. Clinicopathological features of signet-ring cell melanoma.

\begin{tabular}{lllllllll}
\hline $\begin{array}{l}\text { Case } \\
\text { no. }\end{array}$ & $\begin{array}{l}\text { Age, } \\
\text { years }\end{array}$ & Gender & \multicolumn{1}{c}{ Site } & Primary & $\begin{array}{l}\text { S-100 } \\
\text { protein }\end{array}$ & HMB-45 & Vimentin & Author \\
\hline 1 & 35 & Male & Right axillary lymph node & Unknown & + & + & + & Sheibani and Battifora \\
2 & 63 & Female & Inguinal lymph node & Skin & + & + & + & Sheibani and Battifora \\
3 & 57 & Female & Lung & NA & - & + & + & Bonetti et al \\
4 & 55 & Male & Arm (recurrence) & Arm & + & + & + & Nakhleh et al \\
5 & 40 & Male & Thigh (recurrence) & Thigh & + & + & + & Nakhleh et al \\
6 & 80 & Male & Left leg & NA & + & + & + & Al-Talib et al \\
7 & 27 & Male & Multiple metastases & Unknown & + & + & + & Eckert et al \\
8 & 84 & Male & Right forearm & Unknown & + & + & NA & LiVolsi et al \\
9 & 33 & Female & Axillary lymph node & Arm & + & - & NA & LiVolsi et al \\
10 & 85 & Female & Skin and inguinal lymph node & Left foot & + & + & NA & LiVolsi et al \\
11 & 56 & Male & Left ear & NA & + & + & NA & LiVolsi et al \\
12 & 84 & Male & Inguinal lymph node & Righ foot & + & + & + & Tsang et al \\
13 & 55 & Male & Abdomen & Unknown & + & + & + & Won et al \\
14 & 55 & Male & Peritoneal effusion & Abdomen & + & + & + & Niemann et al \\
15 & 72 & Female & Left arm & NA & + & + & + & Breier et al \\
16 & 18 & Female & Inguinal lymph node & NA & NA & + & + & Bastian et al \\
17 & 61 & Female & Right shoulder & Right shoulder & + & - & + & Rutten et al \\
18 & 76 & Male & Anterior chest & Anterior chest & + & + & + & Rutten et al \\
19 & 69 & Male & Left shoulder & Left shoulder & + & - & + & Kacerovska et al \\
20 & 41 & Male & Supraclavicular & Unknown & + & + & NA & Russo et al \\
Present & 68 & Male & Left thigh & Unknown & + & - & + & Ishida et al \\
\hline
\end{tabular}

NA, not available.

Ltd., Newcastle upon Tyne, UK), Melan-A (A103; Novocastra Laboratories, Ltd.), nestin (10C2; Santa Cruz Biotechnology Inc., Santa Cruz, CA, USA), peripherin (PJM50) and vimentin (VIM3B4; both Novocastra Laboratories, Ltd.); and rabbit polyclonal antibodies against S-100 protein (Nichirei Biosciences Inc., Tokyo, Japan) and mTOR (7C10), 4E-BP1 (53H11) and phosphorylated 4E-BP1 (p4E-BP1; Thr 37/46; 236B4) (all Cell Signaling Technology Inc., Danvers, MA, USA).

Results. Histopathological examination of the resected thigh nodule revealed proliferation of sheet-like or variable-sized nests composed of medium-sized, round to oval neoplastic cells from the entire dermis to the superficial subcutis. The majority of the neoplastic cells exhibited slightly eosinophilic cytoplasm and centrally located enlarged nuclei with conspicuous nucleoli and specific cells exhibited melanin pigment within the cytoplasm. In the lower section of the lesion, the neoplastic cells showing signet-ring cell appearance were identified (Fig. 1A). These cells exhibited eccentrically located enlarged nuclei with or without conspicuous nucleoli and abundant pale cytoplasm (Fig. 1A). Melanin pigment was not notably observed in the cytoplasm of the signet-ring cells. Mitotic figures were occasionally identified in the entire lesion (6/10 high-power fields). In addition, no atypical melanocytes were observed in the overlying epidermis. Immunohistochemically, the tumor cells, including signet-ring cells, were diffusely positive for S-100 protein, vimentin and Melan-A (Fig. 1B), but negative for HMB-45, cytokeratin (AE1/AE3 and CAM5.2), nestin, peripherin, $\alpha$-internexin and GFAP. Moreover, mTOR, 4E-BP1 and p4E-BP1 were diffusely expressed in the tumor cells (Fig. 1C).

The sentinel lymph node exhibited a metastatic malignant melanoma with signet-ring cell component (Fig. 2). Immunohistochemical features of the metastatic melanoma were identical to those of the skin.

According to these histopathological and immunohistochemical features, an ultimate diagnosis of signet-ring cell melanoma with sentinel lymph node metastasis was made.

\section{Discussion}

In cutaneous neoplasms, the presence of signet-ring cells have been reported in a variety of neoplasms, including melanocytic nevi, malignant melanoma, squamous and basal cell carcinoma, hidradenoma and malignant lymphoma (5). Ultrastructural examination revealed that this characteristic morphology in signet-ring cell melanoma is often imparted by the intracytoplasmic accumulation of intermediate filaments, particularly vimentin (2). This observation corroborates the immunohistochemical results of the present case report since vimentin was found to be expressed in the cytoplasm of the signet-ring cells, although, ultrastructural examination 

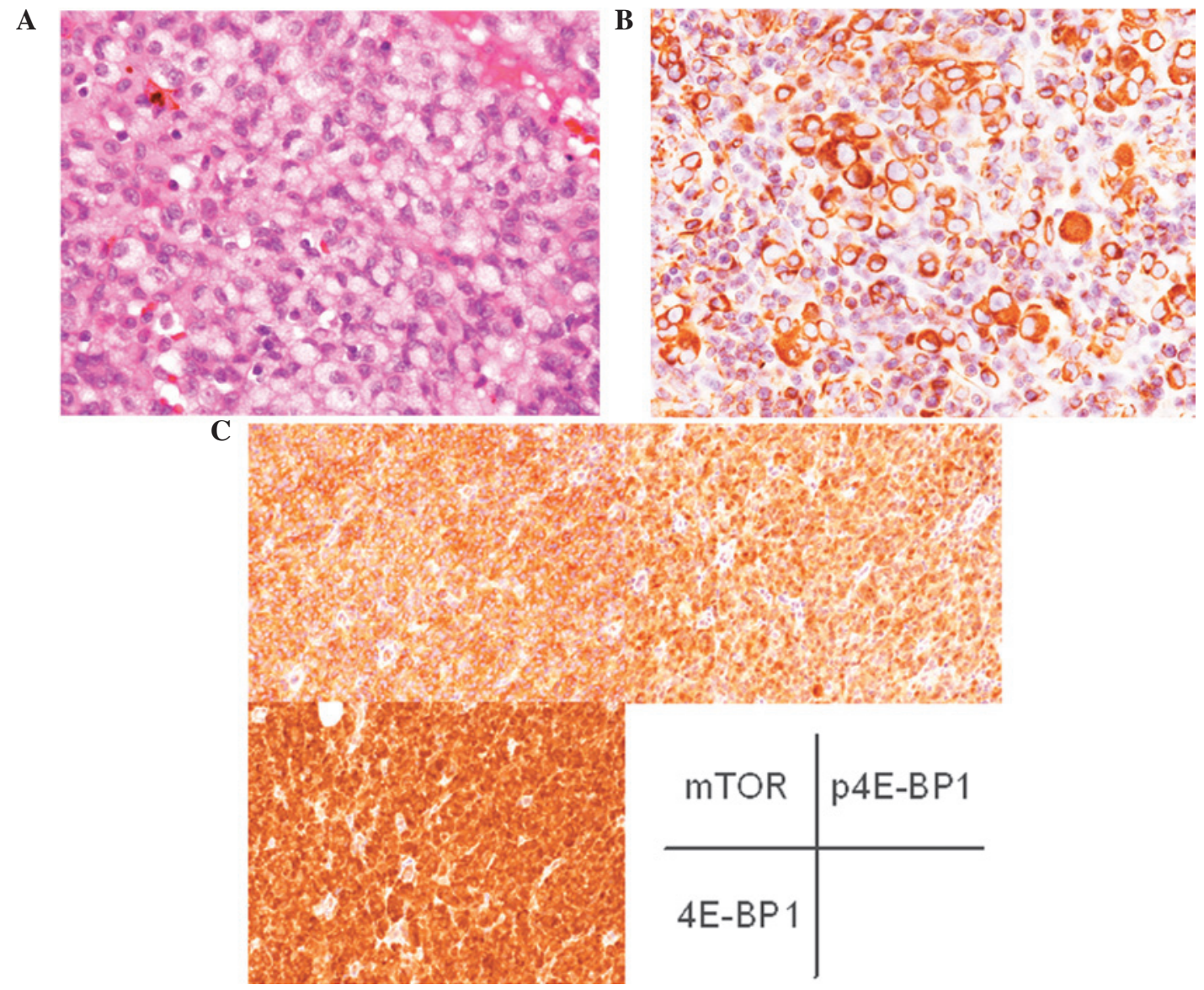

Figure 1. Histopathological and immunohistochemical features of the thigh nodule. (A) Signet-ring cell appearance of the neoplastic cells. The neoplastic cells exhibited eccentrically located enlarged nuclei and abundant pale cytoplasm (hematoxylin and eosin stain; magnification, x400). (B) Vimentin was expressed in the signet-ring cells (magnification, x400). (C) mTOR, 4E-BP1 and p4E-BP1 were diffusely expressed (magnification, x100). mTOR, mammalian target of rapamycin; p4E-BP1, phosphorylated 4E-BP1.

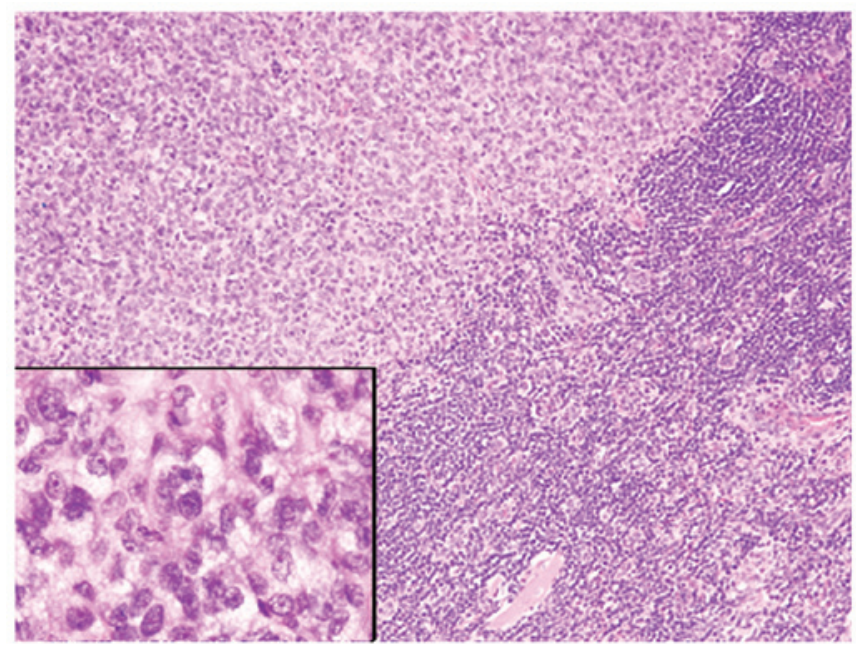

Figure 2. Histopathological features of the sentinel lymph node. Metastatic malignant melanoma was identified. Specific tumor cells showed signet-ring cell appearance as observed in the inset (magnification, $x 400$ ) (hematoxylin and eosin stain; magnification, x100).

was not performed. In addition, analyses of the expression profiles of other intermediate filaments in the signet-ring cell melanoma were not performed. The present case report clearly demonstrated that intermediate filaments, including cytokeratin, peripherin, $\alpha$-internexin, GFAP and nestin, were not expressed in the signet-ring cell melanoma, although, peripherin and nestin are commonly expressed in malignant melanoma (16). These results indicate that intracytoplasmic accumulation of vimentin, but not other types of intermediate filaments, contributes to the development of the characteristic morphology of signet-ring cell melanoma.

Table I summarizes the clinicopathological features of the 20 previously reported cases of signet-ring cell melanoma as well as the present case. This disease commonly affects middle-aged males (average age of 57.8-years and male/female ratio of 14:7), however, young individuals may also be affected (range, 18-85-years-old). Metastatic signet-ring cell melanoma in a patient with an unknown primary tumor has been documented (2) and six cases, including the present case, exhibited no primary sites (Table I). The present case exhibited no in situ component in the overlying epidermis and the patient had no past history of malignant melanoma and tumorous lesions with the exception of the tumor in the thigh, therefore, the cutaneous lesion may not be confirmed as the primary lesion.

Metastatic signet-ring cell melanoma may be a diagnostic issue (4) as it has been reported that signet-ring cells are occasionally present only in metastatic sites and the signet-ring cell melanoma component occasionally lacks melanin pigments in the cytoplasm. Immunohistochemical analyses are useful for generating a correct diagnosis. This type of tumor usually 
shows positive immunoreactivity for melanocytic markers, including S-100 protein, Melan-A and HMB-45. However, it is important to recognize that exceptions to this phenotype exist as $\mathrm{S}-100$ protein-negative (1/20 cases) or HMB-45-negative cases $(4 / 21$ cases) have been documented (Table I) $(2,4)$. Therefore, a combination of these markers is useful for establishing a diagnosis.

In addition, the current case report is the first to analyze the expression profiles of mTOR pathway proteins in signet-ring cell melanoma. mTOR is a central protein involved in carcinogenesis, since it phosphorylates 4E-BP1 which leads to cell proliferation, cell cycle progression and angiogenesis. It has been previously reported that the mTOR pathway is activated in malignant melanomas in contrast to benign melanocytic nevi. Therefore, the mTOR inhibitor is hypothesized to represent a promising therapeutic agent for various types of carcinomas. Specific clinical studies with regard to the mTOR inhibitor have been performed in malignant melanoma (17). The present case study demonstrates that mTOR pathway proteins are activated in signet-ring cell melanoma. Therefore, the mTOR inhibitor may be a potential candidate for the treatment of this type of tumor.

\section{References}

1. Banerjee SS and Harris M: Morphological and immunophenotypic variations in malignant melanoma. Histopathology 36: $387-402,2000$

2. Rütten A, Huschka U, Requena C, Rodríguez-Peralto JL and Requena L: Primary cutaneous signet-ring cell melanoma: a clinico-pathologic and immunohistochemical study of two cases. Am J Dermatopathol 25: 418-422, 2003.

3. Sheibani $\mathrm{K}$ and Battifora $\mathrm{H}$ : Signet-ring cell melanoma. A rare morphologic variant of malignant melanoma. Am J Surg Pathol 12: 28-34, 1988.
4. Kacerovska D, Sokol L, Michal M and Kazakov DV: Primary cutaneous signet-ring cell melanoma with pseudoglandular features, spindle cells and oncocytoid changes. Am J Dermatopathol 31: 81-83, 2009

5. Bastian BC, Kutzner H, Yen Ts and LeBoit PE: Signet-ring cell formation in cutaneous neoplasms. J Am Acad Dermatol 41: 606-613, 1999.

6. Bonetti F, Colombari R, Zamboni G and Chilosi M: Signet ring melanoma, S-100 negative. Am J Surg Pathol 13: 522-523, 1989.

7. Nakhleh RE, Wick MR, Rocamora A, Swanson PE and Dehner LP: Morphologic diversity in malignant melanomas. Am J Clin Pathol 93: 731-740, 1990.

8. al-Talib RK and Theaker JM: Signet-ring cell melanoma: light microscopic, immunohistochemical and ultrastructural features. Histopathology 18: 572-575, 1991.

9. Eckert F, Baricevic B, Landthaler M and Schmid U: Metastatic signet-ring cell melanoma in a patient with an unknown primary tumor. Histologic, immunohistochemical, and ultrastructural findings. J Am Acad Dermatol 26: 870-875, 1992.

10. LiVolsi VA, Brooks JJ, Soslow R, Johnson BL and Elder DE: Signet cell melanocytic lesions. Mod Pathol 5: 515-520, 1992.

11. Tsang WY, Chan JK and Chow LT: Signet-ring cell melanoma mimicking adenocarcinoma. A case report. Acta Cytol 37: 559-562, 1993

12. Won JH, Ahn SK, Lee SH, Lee WS and Kim SC: Signet-ring cell melanoma: poor prognostic factor? Br J Dermatol 131: 135-137, 1994.

13. Niemann TH and Thomas PA: Melanoma with signet-ring cells in a peritoneal effusion. Diagn Cytopathol 12: 241-244, 1995.

14. Breier F, Feldmann R, Fellenz C, Neuhold N and Gschnait F: Primary invasive signet-ring cell melanoma. J Cutan Pathol 26: 533-536, 1999.

15. Russo JJ, Barr KL, Scanlan LZ, Chapman-Fredricks J, Herrera L, Dinges MM and Vincek V: Signet ring cell melanoma, Brenner sign, and elevated vascular endothelial growth factor. J Am Acad Dermatol 65: 444-446, 2011.

16. Brychtova S, Fiuraskova M, Hlobilková A, Brychta T and Hirnak J: Nestin expression in cutaneous melanomas and melanocytic nevi. J Cutan Pathol 34: 370-375, 2007.

17. Dronca RS, Allred JB, Perez DG, et al: Phase II Study of Temozolomide (TMZ) and Everolimus (RAD001) Therapy for Metastatic Melanoma: A North Central Cancer Treatment Group Study, N0675. Am J Clin Oncol: Jan 24, 2013 (Epub ahead of print). 